\title{
Classical fluctuation electrodynamics
}

\author{
A.I.Sokolovsky, A.A.Stupka \\ Dnipropetrovs'k National University, \\ 13 Naukova Str., 49050 Dnipropetrovs'k, Ukraine
}

Received August 3, 2005, in final form November 8, 2005

\begin{abstract}
A system consisting of an equilibrium medium formed by charged particles and electromagnetic field is considered in the classical case at weak interaction between subsystems. The field is described with all the statistical moments of electric and magnetic fields. The moments are reduced description parameters of the herein developed theory based on the Bogolyubov reduced description method of nonequilibrium states. The generalized Bogolyubov condition of the complete correlation weakening between the subsystems is used as a boundary condition to the Liouville equation. Distribution function of the system is calculated up to the third order in electromagnetic interaction. Time equations for the reduced description parameters are written in a compact form using a generating functional for the field moments and a generating functional for field correlations (centered moments, fluctuations). The obtained equations generalize the nonlinear electrodynamics in equilibrium media for the case of fluctuations of electromagnetic field being taken into account.
\end{abstract}

Key words: Bogolyubov reduced description method, complete correlation weakening, equilibrium medium, fluctuation electrodynamics, generating functional

PACS: 05.20.-y, 05.40.-a, 11., 52.40.Db

\section{Introduction}

Almost all the observed phenomena are connected with electromagnetic interactions. Therefore, deep understanding of electromagnetic phenomena and their adequate description will always attract the theoretical thought. The rapid development of quantum optics with special attention to new states of electromagnetic field and the advances of plasma physics have urged the researchers to take into account higher correlation functions of the field (see, for example, [1]). The idea to consolidate the modern directions of nonlinear, stochastic, and fluctuation electrodynamics in the media in a more general theory taking into account all correlations and having a microscopic basis seems to be an important goal. Substantial steps have been made in this way by Klimontovich [2]. However, he studied only binary correlations of the field. Balescu and other representatives of the Brussels school have applied a power- 
ful diagram technique to generalize the equations of nonlinear electrodynamics, but they did not study the own degrees of freedom of the field in detail [3]. Phenomenological nonlinear electrodynamics is based on the idea of expanding the average electric current in powers of small electric and magnetic fields. The coefficients of this expansion (generalized conductivities) remain indefinite in this approach. It is obvious that such material equations do not take field correlations into account.

In the present paper an electromagnetic field in equilibrium medium is described with all the moments of electric and magnetic fields based on the Bogolyubov method of reduced description of nonequilibrium processes [4,5]. The analogous problem for hydrodynamics was considered in [6]. This way the correlation contributions to material equations are studied and microscopic expressions for generalized conductivities are obtained (about these problems see [7]).

\section{Construction of the system distribution function}

We consider an electromagnetic field (f-subsystem) interacting with charged and neutral particles (m-subsystem, medium). The Hamilton function of the system $\mathrm{f}+\mathrm{m}$ in a quasi-relativistic approximation takes the form

$$
\begin{array}{ll}
\hat{H}=\sum_{i} \frac{\left(p_{i}-\frac{e_{i}}{c} \hat{A}\left(x_{i}\right)\right)^{2}}{2 m_{i}}+\hat{H}_{\mathrm{f}}+\hat{H}_{\mathrm{int}}, & \hat{H}_{\mathrm{f}}=\frac{1}{8 \pi} \int \mathrm{d}^{3} x\left(\hat{E}^{2}+\hat{B}^{2}\right) ; \\
\hat{B}(x) \equiv \operatorname{rot} \hat{A}(x), & \hat{E}(x)=-4 \pi c \hat{P}(x) .
\end{array}
$$

Here $\hat{H}_{\mathrm{f}}$ is free electromagnetic field contribution, $\hat{H}_{\text {int }}$ corresponds to a direct interaction between particles which is additional to the interaction via the field. Therefore, we have

$$
\begin{array}{ll}
\hat{H}=\hat{H}_{\mathrm{f}}+\hat{H}_{\mathrm{m}}+\hat{H}_{\mathrm{mf}}, & \hat{H}_{\mathrm{m}} \equiv \sum_{i} \frac{p_{i}^{2}}{2 m_{i}}+\hat{H}_{\mathrm{int}}, \\
\hat{H}_{\mathrm{mf}}=\hat{H}_{1}+\hat{H}_{2}, & \hat{H}_{1} \equiv-\frac{1}{c} \int \mathrm{d} x \hat{A}_{n}(x) \hat{j}_{n}(x), \quad \hat{H}_{2} \equiv \frac{1}{2 c^{2}} \int \mathrm{d} x \hat{\chi}(x) \hat{A}(x)^{2}, \\
\hat{j}_{n}(x) \equiv \sum_{i} \frac{p_{i n}}{m_{i}} e_{i} \delta\left(x-x_{i}\right), & \hat{\chi}(x) \equiv \sum_{i} \frac{e_{i}^{2}}{m_{i}} \delta\left(x-x_{i}\right) .
\end{array}
$$

Here $\hat{H}_{\mathrm{mf}}$ is the interaction between electromagnetic field and particles, $\hat{H}_{m}$ is the Hamilton function of particles. We use the Hamilton gauge of electromagnetic field with $\varphi=0$ and consider vector potential $\hat{A}_{n}(x)$ as a generalized coordinate of the field. $\hat{P}_{n}(x)$ is a corresponding generalized momentum. Hereinafter we mark the functions of the system phase variables with a cap (except the coordinates and momenta of particles). We also assume that some values are vectors but we reflect this in notation only someplace. In this paper the Hamilton technique with the 
following definition of the Poisson brackets is used

$$
\begin{aligned}
\{\hat{f}, \hat{g}\}= & \sum_{i}\left(\frac{\partial \hat{f}}{\partial x_{i n}} \frac{\partial \hat{g}}{\partial p_{i n}}-\frac{\partial \hat{f}}{\partial p_{i n}} \frac{\partial \hat{g}}{\partial x_{i n}}\right) \\
& +\int \mathrm{d}^{3} x\left(\frac{\delta \hat{f}}{\delta \hat{A}_{n}(x)} \frac{\delta \hat{g}}{\delta \hat{P}_{n}(x)}-\frac{\delta \hat{f}}{\delta \hat{P}_{n}(x)} \frac{\delta \hat{g}}{\delta \hat{A}_{n}(x)}\right),
\end{aligned}
$$

( $\hat{f}, \hat{g}$ denote arbitrary functions of phase variables). Therefore, the following formulae for the Poisson brackets of the vector potential and electric field

$$
\left\{\hat{A}_{n}(x), \hat{A}_{l}\left(x^{\prime}\right)\right\}=0, \quad\left\{\hat{E}_{n}(x), \hat{A}_{l}\left(x^{\prime}\right)\right\}=4 \pi c \delta_{n l} \delta\left(x-x^{\prime}\right), \quad\left\{\hat{E}_{n}(x), \hat{E}_{l}\left(x^{\prime}\right)\right\}=0
$$

are true. The relation of electrodynamics

$$
\hat{E}_{n}(x)=-\frac{1}{c} \hat{\dot{A}}_{n}(x)
$$

$(\hat{\dot{f}} \equiv\{\hat{f}, \hat{H}\})$ is obviously true, too. Distribution function $\rho(t)$ of the phase variables of the system satisfies the Liouville equation

$$
\begin{aligned}
& \partial_{t} \rho(t)=\mathbf{L} \rho(t) ; \quad \mathbf{L} \rho(t) \equiv\{\hat{H}, \rho(t)\} \\
& \mathbf{L}=\mathbf{L}_{0}+\mathbf{L}_{\mathrm{mf}}, \quad \mathbf{L}_{0} \equiv \mathbf{L}_{\mathrm{m}}+\mathbf{L}_{\mathrm{f}}, \quad \mathbf{L}_{\mathrm{mf}}=\mathbf{L}_{1}+\mathbf{L}_{2},
\end{aligned}
$$

where contributions to the Liouville operator correspond to contributions to the Hamilton function.

We will study the states of the system for which relations

$$
\hat{H}_{\mathrm{f}} \sim \hat{H}_{\mathrm{m}}, \quad \hat{H}_{\mathrm{f}} \gg \hat{H}_{1}, \hat{H}_{2} ; \quad B(x) \sim E(x)
$$

are valid. Formulae (1), (2) give the following estimates

$$
\begin{aligned}
& \hat{H}_{\mathrm{m}} \sim n V T, \quad \hat{H}_{\mathrm{f}} \sim V E^{2} ; \quad E \sim k A, \quad \omega \sim k c ; \\
& \hat{j} \sim e n v_{T}, \quad \hat{\chi} \sim \frac{e^{2} n}{m} ; \quad \hat{H}_{1} \sim n V T \frac{\Omega}{\omega}, \quad \hat{H}_{2} \sim n V T \frac{\Omega^{2}}{\omega^{2}} .
\end{aligned}
$$

Here $n, T$ are density and temperature of the medium, $\omega, k$ are frequency and wave number of the field, $V$ is volume of the system $f+m$. Characteristic velocity $v_{T}$ and plasma frequency $\Omega$ are given by the expression

$$
v_{T}=\sqrt{\frac{3 T}{m}}, \quad \Omega=\sqrt{\frac{4 \pi e^{2} n}{m}},
$$

( $m$ is the electron mass and $e$ is the module of its charge). Therefore, such relations

$$
\mathbf{L}_{\mathrm{m}} \sim \omega, \quad \sim \mathbf{L}_{\mathrm{f}} \sim \omega, \quad \mathbf{L}_{1} \sim \Omega, \quad \mathbf{L}_{2} \sim \frac{\Omega^{2}}{\omega}
$$


are valid ( $\omega_{\mathrm{m}}$ is a characteristic frequency of medium processes). This allows us to study the system in a perturbation theory in $g$ with estimates

$$
\mathbf{L}_{\mathrm{m}} \sim g^{0}, \quad \mathbf{L}_{\mathrm{f}} \sim \frac{\omega}{\omega_{\mathrm{m}}} g^{0}, \quad \mathbf{L}_{1} \sim g^{1}, \quad \mathbf{L}_{2} \sim \frac{\omega_{\mathrm{m}}}{\omega} g^{2} ; \quad g \equiv \Omega / \omega_{\mathrm{m}} .
$$

Let us describe the electromagnetic field in the equilibrium medium by all the moments of electric $\hat{E}_{n}(x)$ and magnetic $\hat{B}_{n}(x)$ fields. In order to simplify the consideration, we will use cumulative indices $a(b, c), \mu$ and cumulative notation for electromagnetic field

$$
\begin{aligned}
& a \equiv\left(\mu_{1}, \ldots, \mu_{s}\right), \quad \mu \equiv(i, n, x) ; \\
& \hat{\xi}_{\mu} \equiv \hat{\xi}_{i n}(x): \quad \hat{\xi}_{1 n}(x)=\hat{B}_{n}(x), \quad \hat{\xi}_{2 n}(x)=\hat{E}_{n}(x) ; \\
& \hat{\eta}_{a} \equiv \hat{\eta}_{\mu_{1} \ldots \mu_{s}}=\hat{\xi}_{\mu_{1}} \ldots \hat{\xi}_{\mu_{s}} \quad(1 \leqslant s \leqslant \infty) ; \\
& \sum_{a} \equiv \sum_{\mu_{1} \ldots \mu_{s}}, \quad \sum_{\mu} \equiv \sum_{i, n} \int \mathrm{d} x ; \quad \frac{\partial}{\partial \xi_{\mu}} \equiv \frac{\delta}{\delta \xi_{\text {in }}(x)} .
\end{aligned}
$$

In a general case the mentioned description of the field is possible at long times $t \gg \tau_{0}$ where a characteristic time $\tau_{0}$ depends on the initial state $\rho_{0}$ of the system. Our consideration is based on the Bogolyubov idea of the functional hypothesis $[4,5]$

$$
\rho(t) \underset{t \gg \tau_{0}}{\longrightarrow} \rho\left(\eta\left(t, \rho_{0}\right)\right)
$$

where the reduced description parameters $(\mathrm{RDP}) \eta_{a}\left(t, \rho_{0}\right)$ are defined by the formula

$$
\operatorname{Sp} \rho(t) \hat{\eta}_{a} \underset{t \gg \tau_{0}}{\longrightarrow} \eta_{a}\left(t, \rho_{0}\right), \quad \rho_{0} \equiv \rho(t=0),
$$

(Sp denotes the integration over phase variables). The distribution function of the system $\rho(\eta)$ in (13) does not depend on its initial state $\rho_{0}$ and time $t$. For the system under consideration the following relations

$$
\mathbf{L}_{0} \hat{\xi}_{\mu}=-\mathrm{i} \sum_{\mu^{\prime}} c_{\mu \mu^{\prime}} \hat{\xi}_{\mu^{\prime}}, \quad \mathbf{L}_{0} \hat{\eta}_{a}=-\mathrm{i} \sum_{b} c_{a b} \hat{\eta}_{b}
$$

are true because they are a matrix form of the microscopic Maxwell equations for a free electromagnetic field

$$
\mathbf{L}_{0} \hat{E}(x)=-c \operatorname{rot} \hat{B}(x), \quad \mathbf{L}_{0} \hat{B}(x)=c \operatorname{rot} \hat{E}(x) .
$$

Non-zero elements of matrix $c_{\mu \mu^{\prime}}$ are

$$
\begin{aligned}
c_{\mu \mu^{\prime}} & \equiv c_{i n, i^{\prime} n^{\prime}}\left(x, x^{\prime}\right) ; \quad c_{1 n, 2 l}\left(x, x^{\prime}\right)=\mathrm{i} c \varepsilon_{n m l} \frac{\partial \delta\left(x-x^{\prime}\right)}{x_{m}} \\
c_{1 n, 2 l}\left(x, x^{\prime}\right) & =-\mathrm{i} c \varepsilon_{n m l} \frac{\partial \delta\left(x-x^{\prime}\right)}{x_{m}}
\end{aligned}
$$


Matrix $c_{a b}$ has only diagonal elements and, for example,

$$
c_{\mu_{1} \mu_{2}, \mu_{1}^{\prime} \mu_{2}^{\prime}}=c_{\mu_{1} \mu_{1}^{\prime}} \delta_{\mu_{2} \mu_{2}^{\prime}}+\delta_{\mu_{1} \mu_{1}^{\prime}} c_{\mu_{2} \mu_{2}^{\prime}} .
$$

We call a nonequilibrium theory with the property (15) of $\hat{\eta}_{a}$ the PeletminskiiYatsenko model [5].

The RDP's $\eta_{a}\left(t, \rho_{0}\right)$ satisfy the following time equation

$$
\begin{aligned}
\partial_{t} \eta_{a}\left(t, \rho_{0}\right) & =L_{a}\left(\eta\left(t, \rho_{0}\right)\right) \\
L_{a}(\eta) & =\mathrm{i} \sum_{b} c_{a b} \eta_{b}+\tilde{L}_{a}(\eta), \quad \tilde{L}_{a}(\eta) \equiv \operatorname{Sp} \hat{\eta}_{a} \mathbf{L}_{\mathrm{mf}} \rho(\eta) .
\end{aligned}
$$

For the system distribution function $\rho(\eta)$ we have the following equations

$$
\mathbf{L} \rho(\eta)=\sum_{a} \frac{\partial \rho(\eta)}{\partial \eta_{a}} L_{a}(\eta), \quad \operatorname{Sp} \rho(\eta) \hat{\eta}_{a}=\eta_{a}
$$

According to Bogolyubov [4], to find their unique solution one has to add to equations (20) a boundary condition. We shall use the boundary condition of the complete correlation weakening

$$
e^{\tau \mathbf{L}_{0}} \rho(\eta) \underset{t \gg \tau_{0}}{\longrightarrow} e^{\tau \mathbf{L}_{0}} w \rho_{q}(\eta), \quad\left(w \equiv e^{\frac{F-\hat{H}_{m}}{T}}\right),
$$

where $w$ is the Gibbs distribution for the equilibrium medium with temperature $T$, $\rho_{q}(\eta)$ is a quasi-equilibrium distribution function of the field

$$
\rho_{q}(\eta)=\exp \left\{\Omega(\eta)-\sum_{a} Z_{a}(\eta) \hat{\eta}_{a}\right\}, \quad \operatorname{Sp}_{\mathrm{f}} \rho_{q}(\eta) \hat{\eta}_{a}=\eta_{a}, \quad \operatorname{Sp}_{\mathrm{f}} \rho(\eta)=1,
$$

$\left(\mathrm{Sp}_{\mathrm{f}}\right.$ denotes the integration over phase space of the f-subsystem). The second and the third formulae here yield the function $Z_{a}(\eta)$ and $\Omega(\eta)$. The distribution function $\rho_{q}(\eta)$ satisfies the Liouville equation for free subsystem $\mathrm{f}$

$$
\mathbf{L}_{\mathrm{f}} \rho_{q}(\eta)=\sum_{a, b} \frac{\partial \rho_{q}(\eta)}{\partial \eta_{a}} \mathrm{i} c_{a b} \eta_{b}
$$

(see [5]) and has the following property

$$
e^{\tau \mathbf{L}_{0}} \rho_{q}(\eta)=\rho_{q}\left(e^{\mathrm{ic} \tau} \eta\right)
$$

The boundary condition means that the free evolution of the system breaks all the spatial correlations between particles and field because the distance between these subsystems increases. The idea of spatial correlation weakening by means of the free evolution operator $e^{\tau \mathbf{L}_{0}}$ belongs to Bogolyubov [4]. Using the free evolution operator in the boundary condition does not mean that it is correct only approximately. In fact here we do not need a concrete expression for distribution function $\rho_{q}(\eta)$ due to the of relation $\hat{\eta}_{a} \hat{\eta}_{b}=\hat{\eta}_{a \cup b}$ and the second formula in (22). 
It is convenient to rewrite the boundary condition (14) as follows:

$$
\lim _{\tau \rightarrow+\infty} e^{\tau \mathbf{L}_{0}} \rho\left(e^{-\mathrm{i} \mathbf{c} \tau} \eta\right)=w \rho_{q}(\eta)
$$

proceeding from the formula (24). Using the standard procedure [5] we get the following integral equation for the system distribution function $\rho(\eta)$

$$
\rho(\eta)=\rho_{q}(\eta) w+\int_{0}^{+\infty} \mathrm{d} \tau e^{\tau \mathbf{L}_{0}}\left(\mathbf{L}_{\mathrm{mf}} \rho(\eta)-\sum_{\mathbf{a}} \frac{\partial \rho(\eta)}{\partial \eta_{\mathbf{a}}} \tilde{\mathbf{L}}_{\mathbf{a}}(\eta)\right)_{\eta \rightarrow e^{-\mathbf{i} c} \eta} .
$$

For the case of a closed system this equation was first obtained by Peletminskii and Yatsenko [5]. The obtained equation is solvable in a perturbation theory in small parameter $g$ but it is not very convenient to study high order approximations with it. Therefore, let us introduce an auxiliary distribution function

$$
\rho(\eta, \tau)=e^{-\tau \mathbf{L}_{0}} \rho\left(e^{\mathrm{i} \mathbf{c} \tau} \eta\right)
$$

Simple considerations show that it satisfies the following integral equation

$$
\rho(\eta, \tau)=\rho_{q}(\eta) w+\int_{-\infty}^{\tau} \mathrm{d} \tau^{\prime}\left(\mathbf{L}_{\mathrm{mf}}\left(\tau^{\prime}\right) \rho\left(\eta, \tau^{\prime}\right)-\sum_{a} \frac{\partial \rho\left(\eta, \tau^{\prime}\right)}{\partial \eta_{a}} \tilde{L}_{a}\left(\eta, \tau^{\prime}\right)\right)
$$

where

$$
\tilde{L}_{a}(\eta, \tau)=\operatorname{Sp} \hat{\eta}_{a} \mathbf{L}_{\mathrm{mf}}(\tau) \rho(\eta, \tau) ; \quad \mathbf{L}_{\mathrm{mf}}(\tau) \hat{f}=\left\{\hat{H}_{\mathrm{mf}}(\tau), \hat{f}\right\}, \quad \hat{f}(\tau)=e^{-\tau \mathbf{L}_{0}} \hat{f} .
$$

$\hat{f}$ denotes an arbitrary function of the phase variables; therefore, $\hat{f}(\tau)$ gives the corresponding value at the time moment $\tau$ after free evolution. This equation is also solvable in the perturbation theory at a small parameter $g$ introduced in (11)

$$
\begin{aligned}
\rho(\eta, \tau) & =\sum_{s=0}^{\infty} \rho^{(s)}(\eta, \tau), \quad \rho^{(s)}(\eta, \tau) \equiv w R_{s}(\eta, \tau), \\
R_{s}(\eta, \tau) & \sim g^{s}, \quad R_{0}(\eta, \tau)=\varrho_{q}(\eta) w .
\end{aligned}
$$

Moreover, in this paper we restrict ourselves to the consideration of the nonrelativistic approximation taking into account that the value $v_{T} / c$ is an additional small parameter. We shall also assume that the average equilibrium current is equal to zero

$$
\operatorname{Sp}_{\mathrm{m}} w \hat{j}_{n}(x)=0
$$

( $\mathrm{Sp}_{\mathrm{m}}$ denotes the integration over phase space of m-subsystem; $\operatorname{Sp} \hat{A}=\operatorname{Sp}_{\mathrm{m}} \operatorname{Sp}_{\mathrm{f}} \hat{A}$ ).

Let us note that contributions $\hat{H}_{1}$ and $\hat{H}_{2}$ to $\hat{H}_{\mathrm{mf}}$ are of the order $1 / c, 1 / c^{2}$ respectively. There are two origins of $c$ powers in numerators of the considered expressions for $\rho(\eta, \tau)$. The Hamilton functions $\hat{H}_{1}, \hat{H}_{2}$ contribute to $\rho(\eta, \tau)$ through 
the Poisson brackets. One Poisson bracket can give only $c^{1}$ in a numerator because formulae

$$
\left\{\hat{A}_{n}(x), \hat{E}_{l}\left(x^{\prime}\right)\right\}=-4 \pi c \delta_{n l} \delta\left(x-x^{\prime}\right), \quad \partial_{t} \hat{A}_{n}(x, t)=-c \hat{E}_{n}(x, t) .
$$

Therefore, we have to omit a $\hat{H}_{2}$ contribution to the Hamilton function $\hat{H}_{\mathrm{mf}}$ in the considered approximation. Taking into account (32), we can rewrite our typical expression in the perturbation theory as

$$
\begin{aligned}
\int_{-\infty}^{\tau} \mathrm{d} \tau & \left\{\hat{H}_{1}\left(\tau^{\prime}\right), \rho^{(s)}\left(\eta, \tau^{\prime}\right)\right\} f\left(\tau^{\prime}\right) \\
& =\frac{1}{c} \iint_{-\infty}^{\tau} \mathrm{d} x\left\{w R_{s}\left(\eta, \tau^{\prime}\right), \hat{j}_{n}\left(x, \tau^{\prime}\right) \hat{A}_{n}\left(x, \tau^{\prime}\right)\right\} f\left(\tau^{\prime}\right) \\
= & \frac{1}{c} \int_{-\infty}^{\tau} \mathrm{d} \tau^{\prime} \int \mathrm{d} x w\left(-\frac{1}{T}\left\{\hat{H}_{\mathrm{m}}, \hat{j}_{n}\right\} R_{s} \hat{A}_{n}+\left\{R_{s}, j_{n}\right\} \hat{A}_{n}+\left\{R_{s}, \hat{A}_{n}\right\} \hat{j}_{n}\right) f\left(\tau^{\prime}\right) \\
= & \int_{-\infty}^{\tau} \mathrm{d} \tau^{\prime} \int \mathrm{d} x w \mathbf{M}\left(x, \tau^{\prime}\right) R_{s}\left(\eta, \tau^{\prime}\right) f\left(\tau^{\prime}\right)+O\left(v_{T} / c\right) .
\end{aligned}
$$

Here $f(\tau)$ is an arbitrary function. A useful operator in the phase space

$$
\mathbf{M}(x, t) \hat{f} \equiv \hat{j}_{n}(x, t)\left(\frac{1}{c}\left\{\hat{f}, \hat{A}_{n}(x, t)\right\}+\frac{1}{T} \hat{E}_{n}(x, t) \hat{f}\right)
$$

was introduced (we denote operators in the phase space with bold letters).

Applying the mentioned ideas, we obtain the following expressions for functions $R_{s}(\eta, \tau)$ defined by $(30)$

$$
\begin{aligned}
R_{1}(\eta, \tau)= & \int_{-\infty}^{\tau} \mathrm{d} \tau^{\prime} \int \mathrm{d} x \mathbf{M}\left(x, \tau^{\prime}\right) \rho_{q}(\eta), \\
R_{2}(\eta, \tau)= & \int_{-\infty}^{\tau} \mathrm{d} \tau^{\prime} \int \mathrm{d} x\left(\mathbf{M}\left(x, \tau^{\prime}\right) R_{1}\left(\eta, \tau^{\prime}\right)-\sum_{a} \frac{\partial \rho_{q}}{\partial \eta_{a}} \operatorname{Sp} \hat{\eta}_{a} w \mathbf{M}\left(x, \tau^{\prime}\right) R_{1}\left(\eta, \tau^{\prime}\right)\right) \\
R_{3}(\eta, \tau)= & \int_{-\infty}^{\tau} \mathrm{d} \tau^{\prime} \int \mathrm{d} x\left(\mathbf{M}\left(x, \tau^{\prime}\right) R_{2}\left(\eta, \tau^{\prime}\right)-\sum_{a} \frac{\partial \rho_{q}}{\partial \eta_{a}} \operatorname{Sp} \hat{\eta}_{a} w \mathbf{M}\left(x, \tau^{\prime}\right) R_{2}\left(\eta, \tau^{\prime}\right)\right. \\
& \left.-\sum_{a} \frac{\partial R_{1}\left(\eta, \tau^{\prime}\right)}{\partial \eta_{a}} \operatorname{Sp} \hat{\eta}_{a} w \mathbf{M}\left(x, \tau^{\prime}\right) R_{1}\left(\eta, \tau^{\prime}\right)\right)
\end{aligned}
$$

(for simplicity we omit here and further the estimations $O\left(v_{T} / c\right)$ ). 


\section{Equations for the generating functional}

In order to make the obtained result more visible, let us introduce the generating functional (GF) $F(\eta, u)$ for all the moments of electromagnetic field [6]

$$
F(\eta, u)=1+\sum_{s=1}^{\infty} \frac{1}{s !} \sum_{\mu_{1} \ldots \mu_{s}} u_{\mu_{1}} \ldots u_{\mu_{s}} \eta_{\mu_{1} \ldots \mu_{s}}
$$

where $u_{\mu} \equiv u_{i n}(x)$ are auxiliary functions. Formulae (12), (20), (22) show that the following relations

$$
\begin{aligned}
\hat{F}(u) & \equiv F(\hat{\eta}, u)=\sum_{a} \frac{\partial F(\eta, u)}{\partial \eta_{a}} \hat{\eta}_{a}=\exp \sum_{\mu} u_{\mu} \hat{\xi}_{\mu} ; \\
F(\eta, u) & =\operatorname{Sp} \rho(\eta) \hat{F}(u)=\operatorname{Sp} \rho_{q}(\eta) \hat{F}(u)
\end{aligned}
$$

are true.

It is easy to derive a closed time equation for $F\left(\eta(t), u\right.$ ) (we omit $\rho_{0}$ from $\eta_{a}\left(t, \rho_{0}\right)$ for simplicity). Statistical operator $\rho(\eta(t))$ satisfies the Liouville equation (6) and therefore we have

$$
\partial_{t} F(\eta(t), u)=\operatorname{Sp} \hat{F}(u) \mathbf{L} \rho(\eta(t))=\sum_{\mu} u_{\mu} \operatorname{Sp} \rho(\eta(t)) \hat{F}(u) \hat{\dot{\xi}}_{\mu}
$$

Value $\hat{\dot{\xi}}_{\mu}=-\mathbf{L} \hat{\xi}_{\mu}$ is given by the microscopic Maxwell equations

$$
\hat{\dot{\xi}}_{\mu}=\mathrm{i} \sum_{\mu^{\prime}} c_{\mu \mu^{\prime}} \hat{\xi}_{\mu^{\prime}}-4 \pi \hat{j}_{\mu}-\mathbf{L}_{\mathbf{2}} \hat{\xi}_{\mu} ; \quad \hat{j}_{\mu} \equiv \hat{j}_{i n}(x)=\delta_{i 2} \hat{j}_{n}(x)
$$

(see (6), (15), (16)). Here the term $\mathbf{L}_{2} \hat{\xi}_{\mu} \sim 1 / c$ must be omitted in the considered approximation.

Note that the calculation of the trace $\mathrm{Sp}_{m}$ over the phase space of the medium gives a formula

$$
\operatorname{Sp} \rho(\eta) \hat{F}(u) \hat{j}_{n}(x) \equiv \operatorname{Sp}_{\mathrm{f}} \rho_{q}(\eta) \hat{F}(u) I_{n}(x, u, \hat{\xi})
$$

with a certain function of a microscopic electromagnetic field $I_{n}(x, \hat{\xi})$. Therefore, using (38)-(40), we have

$$
\partial_{t} F(\eta(t), u)=\sum_{\mu} u_{\mu} \operatorname{Sp} \rho_{q}(\eta(t)) \hat{F}(u) L_{\mu}(u, \hat{\xi}),
$$

where the notation

$$
L_{\mu}(u, \xi)=\mathrm{i} \sum_{\mu^{\prime}} c_{\mu \mu^{\prime}} \xi_{\mu^{\prime}}-4 \pi I_{\mu}(u, \xi), \quad I_{\mu}(u, \xi) \equiv I_{i n}(x, u, \xi)=\delta_{i 2} I_{n}(x, u, \xi)
$$


is introduced. The RHS of equation (41) for $F(\eta(t), u)$ can be expressed through $F(\eta(t), u)$ using the formula

$$
\hat{F}(u) f(\hat{\xi})=\left.\hat{F}\left(u+\frac{\partial}{\partial \xi}\right) f(\xi)\right|_{\xi \rightarrow 0}
$$

which follows from (37). Really, (37), (43) give the necessary closed equation for the generating functional $F(\eta(t), u)$

$$
\partial_{t} F(\eta(t), u)=\left.F\left(\eta(t), u+\frac{\partial}{\partial \xi}\right) \sum_{\mu} u_{\mu} L_{\mu}(u, \xi)\right|_{\xi \rightarrow 0}
$$

This equation is the equation of fluctuation electrodynamics (FED) in the equilibrium medium, i.e. the equation for all the moments of electromagnetic field.

Instead of the GF for moments $F(\eta, u)$ a GF $G(g, u)$ for correlations (fluctuations, centered moments) $g_{a} \equiv g_{\mu_{1} \ldots \mu_{s}}$ of the field can be introduced [6] so that

$$
G(g, u)=\sum_{s=2}^{\infty} \frac{1}{s !} \sum_{\mu_{1} \ldots \mu_{s}} u_{\mu_{1}} \ldots u_{\mu_{s}} g_{\mu_{1} \ldots \mu_{s}}, \quad F(\eta, u)=\exp \left(\sum_{\mu} u_{\mu} \xi_{\mu}+G(g, u)\right)
$$

Simple calculation based on the formula

$$
\left.F\left(\eta(t), u+\frac{\partial}{\partial \xi}\right) f(\xi)\right|_{\xi \rightarrow 0}=\left.e^{\sum_{\mu}^{\mu} \xi_{\mu}(t) u_{\mu}} e^{G\left(g(t), u+\frac{\partial}{\partial \xi}\right)} f(\xi)\right|_{\xi \rightarrow \xi(t)}
$$

gives the following equations for an average value of electromagnetic field and its correlations

$$
\begin{aligned}
\partial_{t} \xi_{\mu}(t) & =\left.e^{G\left(g, \frac{\partial}{\partial \xi}\right)} L_{\mu}(u=0, \xi)\right|_{\xi \rightarrow \xi(t), g \rightarrow g(t)} \\
\partial_{t} G(g(t), u) & =\sum_{\mu} u_{\mu}\left\{e^{G\left(g, u+\frac{\partial}{\partial \xi}\right)-G(g, u)} L_{\mu}(u, \xi)-e^{G\left(g, \frac{\partial}{\partial \xi}\right)} L_{\mu}(u=0, \xi)\right\}_{\xi \rightarrow \xi(t), g \rightarrow g(t)}
\end{aligned}
$$

(compare with [6]). Contribution to (47) from linear in $\hat{\xi}_{\mu}$ terms from (42) (contribution of free electromagnetic field) can be easily calculated using a method of differential equation. Really, function

$$
f_{\mu}(\lambda) \equiv e^{\lambda G\left(g, u+\frac{\partial}{\partial \xi}\right)} \xi_{\mu}
$$

has a derivative

$$
\dot{f}_{\mu}(\lambda) \equiv e^{\lambda G\left(g, u+\frac{\partial}{\partial \xi}\right)}\left(G(g, u)+\sum_{s=1}^{\infty} \frac{1}{s !} \sum_{\mu_{1} \ldots \mu_{s}} G_{\mu_{1} \ldots \mu_{s}}(g, u) \frac{\partial^{s}}{\partial \xi_{\mu_{1}} \ldots \partial \xi_{\mu_{s}}}\right) \xi_{\mu}
$$

where

$$
G_{\mu_{1} \ldots \mu_{s}}(g, u)=\frac{\partial^{s} G(g, u)}{\partial u_{\mu_{1}} \ldots \partial u_{\mu_{s}}}
$$


Therefore, it satisfies the following differential equation

$$
\dot{f}_{\mu}(\lambda)=G(g, u) f_{\mu}(\lambda)+G_{\mu}(g, u) e^{\lambda G(g, u)}, \quad f_{\mu}(0)=\xi_{\mu} .
$$

Solution of this equation leads to identity

$$
e^{G\left(g, u+\frac{\partial}{\partial \xi}\right)-G(g, u)} \xi_{\mu}=\xi_{\mu}+G_{\mu}(g, u) .
$$

So, equation (47) can be rewritten in the form

$$
\begin{aligned}
& \partial_{t} \xi_{\mu}(t)=\mathrm{i} \sum_{\mu^{\prime}} c_{\mu \mu^{\prime}} \xi_{\mu^{\prime}}(t)-4 \pi j_{\mu}(\xi(t), g(t)) \\
& \partial_{t} G(g(t), u)=\mathrm{i} \sum_{\mu \mu^{\prime}} c_{\mu \mu^{\prime}} G_{\mu^{\prime}}(g(t), u) \\
& \quad+\sum_{\mu} u_{\mu}\left\{e^{G\left(g, u+\frac{\partial}{\partial \xi}\right)-G(g, u)} I_{\mu}(u, \xi)-e^{G\left(g, \frac{\partial}{\partial \xi}\right)} I_{\mu}(u=0, \xi)\right\}_{\xi \rightarrow \xi(t), g \rightarrow g(t)}
\end{aligned}
$$

where $I_{\mu}(u, \xi)$ is expressed through $I_{n}(x, u, \xi)$ by the formula (42) and

$$
j_{\mu}(\xi, g) \equiv j_{i n}(x, \xi, g)=\delta_{i 2} j_{n}(x, \xi, g), \quad j_{n}(x, \xi, g)=e^{G\left(g, \frac{\partial}{\partial \xi}\right)} I_{n}(x, u=0, \xi) .
$$

According to (12), (15), (16) the first formula (52) gives the Maxwell equations; therefore, $j_{n}(x, \xi, g)$ is the average electric current in the system.

Equations (52) are equations of the FED in the terms of average electromagnetic field $\xi_{\mu}(t)$ and its correlations (fluctuations, centered moments) $g_{\mu_{1} \ldots \mu_{s}}(t)$. These equations are completely defined by function $I_{n}(x, u, \xi)$ which is introduced by the formula (40). Therefore, the last step of our investigation is to calculate this function. A typical value to be calculated is

$$
\begin{aligned}
\operatorname{Sp} w \hat{F} \hat{j}_{n}(x) \mathbf{M}\left(x^{\prime}, \tau\right) R_{s}= & \operatorname{Sp} w \hat{j}_{n}(x) \hat{j}_{l}\left(x^{\prime}, \tau\right) \\
& \times R_{s}\left(\frac{1}{c}\left\{\hat{A}_{l}\left(x^{\prime}, \tau\right), \hat{F}\right\}+\frac{1}{T} \hat{E}_{l}\left(x^{\prime}, \tau\right) \hat{F}\right) \\
= & \frac{1}{T} \operatorname{Sp} w \hat{j}_{n}(x) \hat{j}_{l}\left(x^{\prime}, \tau\right) R_{s} \alpha_{l}\left(x^{\prime}, \tau, \hat{\xi}+4 \pi T u\right) \hat{F}
\end{aligned}
$$

where

$$
\begin{array}{rlrl}
\alpha_{n}(x, \tau, u) & \equiv \sum_{i} \int \mathrm{d} x^{\prime} \theta_{i n l}\left(x-x^{\prime}, \tau\right) u_{i l}\left(x^{\prime}\right), & \\
\theta_{1 n l}(x, t) & \equiv \lambda_{n l}(x, t), & & \theta_{2 n l}(x, t) \equiv \mu_{n l}(x, t) ; \\
\lambda_{n l}(k, t) & =\mathrm{i} \varepsilon_{n l m} \tilde{k}_{m} \sin \omega_{k} t, & & \mu_{n l}(k, t)=\tilde{k}_{n} \tilde{k}_{l}+\tilde{\delta}_{n l} \cos \omega_{k} t, \\
\tilde{k}_{n} & \equiv k_{n} / k, & \tilde{\delta}_{n l} \equiv \delta_{n l}-\tilde{k}_{n} \tilde{k}_{l} .
\end{array}
$$


Relations (54) also contain the solutions of equations for free electromagnetic field

$$
\begin{aligned}
& \hat{A}_{n}(x, t)=e^{-t \mathbf{L}_{0}} \hat{A}_{n}(x)=\int \mathrm{d} x^{\prime}\left(\mu_{n l}\left(x-x^{\prime}, t\right) \hat{A}_{l}\left(x^{\prime}\right)+\nu_{n l}\left(x-x^{\prime}, t\right) \hat{E}_{l}\left(x^{\prime}\right)\right), \\
& \hat{E}_{n}(x, t)=e^{-t \mathbf{L}_{0}} \hat{E}_{n}(x)=\int \mathrm{d} x^{\prime}\left(\lambda_{n l}\left(x-x^{\prime}, t\right) \hat{B}_{l}\left(x^{\prime}\right)+\mu_{n l}\left(x-x^{\prime}, t\right) \hat{E}_{l}\left(x^{\prime}\right)\right), \\
& \nu_{n l}(k, t)=-\tilde{k}_{n} \tilde{k}_{l} c t-\tilde{\delta}_{n l} \frac{\sin \omega_{k} t}{k}
\end{aligned}
$$

which have the following properties

$$
\begin{aligned}
& \hat{E}_{n}(x, t)=\alpha_{n}(x, t, \hat{\xi}), \quad\left\{\hat{A}_{n}(x, t), \hat{F}(u)\right\}=4 \pi c \alpha_{n}(x, t, u) \hat{F}(u), \\
& \left\{\hat{A}_{n}(x, t), \hat{\xi}_{i l}\left(x^{\prime}, t^{\prime}\right)\right\}=4 \pi c \theta_{\text {inl }}\left(x-x^{\prime}, t-t^{\prime}\right) .
\end{aligned}
$$

From the obtained results one can see that

$$
I_{n}(x, u, \xi)=I_{n}(x, u=0, \xi+4 \pi T u),
$$

where function $I_{n}(x, u=0, \xi)$ is given by formulae:

$$
\begin{aligned}
& I_{n}(x, u=0, \xi)=\sum_{s=2}^{\infty} I_{n}^{(s)}(x, u=0, \xi) \\
& I_{n}^{(2)}(x, u=0, \xi)=\frac{1}{T} \int_{-\infty}^{0} \mathrm{~d} \tau_{1} \int \mathrm{d} x_{1} I_{n l}\left(x_{1}-x, \tau_{1}\right) \alpha_{l}\left(x_{1}, \tau_{1}, \xi\right), \\
& I_{n}^{(3)}(x, u=0, \xi)=\frac{1}{T^{2}} \int_{-\infty}^{0} \mathrm{~d} \tau_{1} \int \mathrm{d} x_{1} \int_{-\infty}^{\tau_{1}} \mathrm{~d} \tau_{2} \int \mathrm{d} x_{2} I_{n l m}\left(x_{1}-x, \tau_{1} ; x_{2}-x, \tau_{2}\right) \\
& \times\left[\alpha_{l}\left(x_{1}, \tau_{1}, \xi\right) \alpha_{m}\left(x_{2}, \tau_{2}, \xi\right)+4 \pi T \mu_{l m}\left(x_{1}-x_{2}, \tau_{1}-\tau_{2}\right)\right], \\
& I_{n}^{(4)}(x, u=0, \xi)=\frac{1}{T^{3}} \int_{-\infty}^{0} \mathrm{~d} \tau_{1} \int \mathrm{d} x_{1} \int_{-\infty}^{\tau_{1}} \mathrm{~d} \tau_{2} \int \mathrm{d} x_{2} \int_{-\infty}^{\tau_{2}} \mathrm{~d} \tau_{3} \int \mathrm{d} x_{3} \\
& \times I_{n l m s}\left(x_{1}-x, \tau_{1} ; x_{2}-x, \tau_{2} ; x_{3}-x, \tau_{3}\right)\left[\alpha_{l}\left(x_{1}, \tau_{1}, \xi\right) \alpha_{m}\left(x_{2}, \tau_{2}, \xi\right) \alpha_{s}\left(x_{3}, \tau_{3}, \xi\right)\right. \\
& +4 \pi T \alpha_{m}\left(x_{2}, \tau_{2}, \xi\right) \mu_{l s}\left(x_{1}-x_{3}, \tau_{1}-\tau_{3}\right)+4 \pi T \alpha_{l}\left(x_{1}, \tau_{1}, \xi\right) \mu_{m s}\left(x_{2}-x_{3}, \tau_{2}-\tau_{3}\right) \\
& \left.+4 \pi T \alpha_{s}\left(x_{3}, \tau_{3}, \xi\right) \mu_{l m}\left(x_{1}-x_{2}, \tau_{1}-\tau_{2}\right)\right] .
\end{aligned}
$$

Here correlation functions of currents $\hat{j}_{n}(x, t)=e^{-t \mathbf{L}_{0}} \hat{j}_{n}(x)$

$$
\begin{aligned}
I_{n l}\left(x_{1}, \tau_{1}\right)= & \operatorname{Sp}_{\mathrm{m}} w \hat{j}_{n}(0) \hat{j}_{l}\left(x_{1}, \tau_{1}\right), \\
I_{n l m}\left(x_{1}, \tau_{1} ; x_{2}, \tau_{2}\right)= & \operatorname{Sp}_{\mathrm{m}} w \hat{j}_{n}(0) \hat{j}_{l}\left(x_{1}, \tau_{1}\right) \hat{j}_{m}\left(x_{2}, \tau_{2}\right), \\
I_{n l s m}\left(x_{1}, \tau_{1} ; x_{2}, \tau_{2} ; x_{3}, \tau_{3}\right)= & \operatorname{Spm}_{\mathrm{m}} w \hat{j}_{n}(0) \hat{j}_{l}\left(x_{1}, \tau_{1}\right) \hat{j}_{m}\left(x_{2}, \tau_{2}\right) \hat{j}_{s}\left(x_{3}, \tau_{3}\right) \\
& -\operatorname{Sp}_{\mathrm{m}} w \hat{j}_{n}(0) \hat{j}_{l}\left(x_{1}, \tau_{1}\right) \operatorname{Sp}_{\mathrm{m}} w \hat{j}_{m}\left(x_{2}, \tau_{2}\right) \hat{j}_{s}\left(x_{3}, \tau_{3}\right) \\
& -\operatorname{Sp}_{\mathrm{m}} w \hat{j}_{n}(0) \hat{j}_{m}\left(x_{2}, \tau_{2}\right) \operatorname{Sp}_{\mathrm{m}} w \hat{j}_{l}\left(x_{1}, \tau_{1}\right) \hat{j}_{s}\left(x_{3}, \tau_{3}\right) \\
& -\operatorname{Sp}_{\mathrm{m}} w \hat{j}_{n}(0) \hat{j}_{s}\left(x_{3}, \tau_{3}\right) \operatorname{Sp}_{\mathrm{m}} w \hat{j}_{l}\left(x_{1}, \tau_{1}\right) \hat{j}_{m}\left(x_{2}, \tau_{2}\right)
\end{aligned}
$$


are introduced. In the considered approximation (59) the function $I_{\mu}(u=0, \xi)$ is given by the formula

$$
\begin{aligned}
I_{\mu}(u=0, \xi)= & S_{\mu}+\sum_{\mu_{1}} \sigma_{\mu, \mu_{1}} \xi_{\mu_{1}}+\sum_{\mu_{1} \mu_{2}} \sigma_{\mu_{, \mu_{1} \mu_{2}}} \xi_{\mu_{1}} \xi_{\mu_{2}} \\
& +\sum_{\mu_{1} \mu_{2} \mu_{3}} \sigma_{\mu, \mu_{1} \mu_{2} \mu_{3}} \xi_{\mu_{1}} \xi_{\mu_{2}} \xi_{\mu_{3}}+O\left(g^{5}\right),
\end{aligned}
$$

where according to (12) and (42) the values $S_{\mu}, \sigma_{\mu, \mu_{1}}, \sigma_{\mu, \mu_{1} \mu_{2}}, \sigma_{\mu, \mu_{1} \mu_{2} \mu_{3}}$ have the structure

$$
\begin{aligned}
& S_{\mu} \equiv S_{i n}(x)=\delta_{i 2} S_{n}, \quad \sigma_{\mu \mu_{1}} \equiv \sigma_{i n, i_{1} n_{1}}\left(x, x_{1}\right)=\delta_{i 2} \sigma_{n, i_{1} n_{1}}\left(x-x_{1}\right), \\
& \sigma_{\mu, \mu_{1} \mu_{2}} \equiv \sigma_{i n, i_{1} n_{1} i_{2} n_{2}}\left(x, x_{1}, x_{2}\right)=\delta_{i 2} \sigma_{n, i_{1} n_{1} i_{2} n_{2}}\left(x-x_{1}, x-x_{2}\right), \ldots
\end{aligned}
$$

For all of them we obtain concrete expressions

$$
\begin{aligned}
& S_{n}=\frac{4 \pi}{T} \int_{-\infty}^{0} \mathrm{~d} \tau \int \mathrm{d} x \int_{-\infty}^{\tau} \mathrm{d} \tau^{\prime} \int \mathrm{d} x^{\prime} I_{n l m}\left(x, \tau ; x^{\prime}, \tau^{\prime}\right) \mu_{l m}\left(x-x^{\prime}, \tau-\tau^{\prime}\right)+O\left(g^{5}\right), \\
& \sigma_{n, i l}\left(x-x^{\prime}\right)=\frac{1}{T} \int_{-\infty}^{0} \mathrm{~d} \tau \int \mathrm{d} x^{\prime \prime} I_{n m}\left(x-x^{\prime \prime}, \tau\right) \lambda_{i m l}\left(x^{\prime \prime}-x^{\prime}, \tau\right)+O\left(g^{4}\right), \ldots
\end{aligned}
$$

Formulae (58), (61) make it possible to calculate the values entering the equations of FED (52). This can be done using the differential equation method (see (50)) for functions

$$
f_{\mu_{1} \ldots \mu_{s}}(\lambda)=e^{\lambda G\left(g, u+\frac{\partial}{\partial \xi}\right)} \xi_{\mu_{1}} \ldots \xi_{\mu_{s}}
$$

which leads to a chain of equations of the first order. This chain can be solved successively starting from equation $(50)$ for $f_{\mu_{1}}(\lambda)$. This way we obtain

$$
\begin{gathered}
e^{G\left(g, u+\frac{\partial}{\partial \xi}\right)-G(g, u)} \xi_{1} \xi_{2}=\xi_{1} \xi_{2}+G_{12}+\xi_{1} G_{2}+\xi_{2} G_{1}+G_{1} G_{2} \\
e^{G\left(g, u+\frac{\partial}{\partial \xi}\right)-G(g, u)} \xi_{1} \xi_{2} \xi_{3}=\xi_{1} \xi_{2} \xi_{3}+G_{123}+\left[G_{12}\left(\frac{1}{2} G_{3}+\xi_{3}\right)+c . p .\right] \\
+\left[G_{3}\left(\xi_{1} \xi_{2}+\frac{1}{2} G_{12}+\frac{1}{2} \xi_{1} G_{2}+\frac{1}{2} \xi_{2} G_{1}+\frac{1}{3} G_{1} G_{2}\right)+c . p .\right],
\end{gathered}
$$

where for simplicity a highly reduced notation

$$
\xi_{i} \equiv \xi_{\mu_{i}}, \quad G_{1 \ldots s} \equiv G_{\mu_{1} \ldots \mu_{s}}
$$

is used.

We will not substitute expressions (65) into equations (52) completely, and restrict ourselves to a final formula for average current in the system $j_{\mu}(\xi, g)$

$$
\begin{aligned}
j_{\mu}(\xi, g)= & S_{\mu}+\sum_{\mu_{1}} \sigma_{\mu, \mu_{1}} \xi_{\mu_{1}}+\sum_{\mu_{1} \mu_{2}} \sigma_{\mu_{\mu} \mu_{1} \mu_{2}}\left(g_{\mu_{1} \mu_{2}}+\xi_{\mu_{1}} \xi_{\mu_{2}}\right) \\
& +\sum_{\mu_{1} \mu_{2} \mu_{3}} \sigma_{\mu, \mu_{1} \mu_{2} \mu_{3}}\left(3 g_{\mu_{1} \mu_{2}} \xi_{\mu_{3}}+g_{\mu_{1} \mu_{2} \mu_{3}}+\xi_{\mu_{1}} \xi_{\mu_{2}} \xi_{\mu_{3}}\right)+O\left(g^{5}\right) .
\end{aligned}
$$


This formula is a material equation for the FED. Values $\sigma_{\mu, \mu_{1}}, \sigma_{\mu, \mu_{1} \mu_{2}}, \sigma_{\mu, \mu_{1} \mu_{2} \mu_{3}}$ can be called generalized conductivities. According to (62) they take into account a spatial dispersion. In equilibrium, the electric current in the considered system must vanish

$$
j_{\mu}\left(\xi=0, g^{\mathrm{eq}}\right)=0, \quad\left(\xi^{\mathrm{eq}}=0\right) .
$$

Therefore, the material equation (67) can be rewritten in the form

$$
\begin{aligned}
j_{\mu}(\xi, g)= & \sum_{\mu_{1}} \sigma_{\mu_{\mu} \mu_{1}} \xi_{\mu_{1}}+\sum_{\mu_{1} \mu_{2}} \sigma_{\mu_{\mu} \mu_{1} \mu_{2}}\left(\delta g_{\mu_{1} \mu_{2}}+\xi_{\mu_{1}} \xi_{\mu_{2}}\right) \\
& +\sum_{\mu_{1} \mu_{2} \mu_{3}} \sigma_{\mu_{\mu_{1} \mu_{2} \mu_{3}}}\left(3 g_{\mu_{1} \mu_{2}} \xi_{\mu_{3}}+\delta g_{\mu_{1} \mu_{2} \mu_{3}}+\xi_{\mu_{1}} \xi_{\mu_{2}} \xi_{\mu_{3}}\right)+O\left(g^{5}\right)
\end{aligned}
$$

where

$$
\delta g_{\mu_{1} \ldots \mu_{s}}=g_{\mu_{1} \ldots \mu_{s}}-g_{\mu_{1} \ldots \mu_{s}}^{\mathrm{eq}} .
$$

Let us compare the developed theory with the theories having a truncated set of nonequilibrium correlations as independent variables. In usual nonlinear electrodynamics (NED) correlations $g_{\mu_{1} \ldots \mu_{s}}$ should be functions of electromagnetic field $g_{\mu_{1} \ldots \mu_{s}}(\xi)$ and a function $j_{0 \mu}(\xi) \equiv j_{\mu}\left(\xi, g_{2}(\xi), g_{3}(\xi) \ldots\right)$ is material equation of the NED (we use a more detailed notation $j_{\mu}\left(\xi, g_{2}, g_{3}, \ldots\right) \equiv j_{\mu}(\xi, g)$ ). Time equations for all correlations (52) should be satisfied with functions $g_{\mu_{1} \ldots \mu_{s}}(\xi(t))(2 \leqslant s<\infty)$.

In FED taking into account only binary correlations $g_{\mu_{1} \mu_{2}}\left(\mathrm{FED}_{2}\right)$ triple and more complicated correlations should be functions of electromagnetic field and binary correlations $g_{\mu_{1} \ldots \mu_{s}}\left(\xi, g_{2}\right)(3 \leqslant s<\infty)$. The material equation of the $\mathrm{FED}_{2}$ in the terms of function (67) is given by formula $j_{1 \mu}\left(\xi, g_{2}\right) \equiv j_{\mu}\left(\xi, g_{2}, g_{3}\left(\xi, g_{2}\right), \ldots\right)$. Time equations for triple and more complicated correlations (52) should be satisfied with functions $g_{\mu_{1} \ldots \mu_{s}}\left(\xi, g_{2}\right)(3 \leqslant s<\infty)$.

Let us truncate the equations (47) of the FED taking into account only binary correlations and construct the corresponding equations of the $\mathrm{FED}_{2}$. Note that the $\mathrm{FED}_{2}$ which describes the electromagnetic field with $\xi_{\mu}$ and $g_{\mu_{1} \mu_{2}}$ can be built based on our equations (19), (22), (26) with operator of RDP's $\hat{\eta}_{a}=\hat{\xi}_{\mu}, \hat{\xi}_{\mu_{1}} \hat{\xi}_{\mu_{2}}$ (see [8,9]). In this case $\rho_{q}$ is a Gauss distribution

$$
\rho_{q}=e^{\Omega-\sum_{\mu} Z_{\mu} \hat{\xi}_{\mu}-\sum_{\mu \mu^{\prime}} Z_{\mu \mu^{\prime}} \hat{\xi}_{\mu} \hat{\xi}_{\mu^{\prime}}}
$$

and averages $\operatorname{Sp} \rho_{q} \hat{\xi}_{\mu_{1}} \ldots \hat{\xi}_{\mu_{s}}$ can be calculated in the terms of $\xi_{\mu}$ and $g_{\mu \mu^{\prime}}$ using the generating functional

$$
F_{q}(u)=\operatorname{Sp}_{\mathrm{f}} \rho_{q} \hat{F}(u)=e^{\sum_{\mu} u_{\mu} \xi_{\mu}+\frac{1}{2} \sum_{\mu \mu^{\prime}} u_{\mu} u_{\mu^{\prime}} g_{\mu \mu^{\prime}}} .
$$

In order to compare FED and $\mathrm{FED}_{2}$ we need $g_{\mu_{1} \ldots \mu_{s}}\left(\xi, g_{2}\right)$ which can be obtained with a generating functional method. According to (30), (31), (37), (45) and using 
the reduced notation (66) we have

$$
\begin{aligned}
G(u)= & \ln F(u)=\ln \operatorname{Sp} \rho(\eta) \hat{F}(u)=\ln \operatorname{Sp}\left(\rho_{q} w+\rho^{(1)}+\rho^{(2)}+\rho^{(3)}+O\left(g^{4}\right)\right) \hat{F}(u) \\
= & \ln F_{q}(u)+F_{q}(u)^{-1} \operatorname{Sp}\left(\rho^{(2)}+\rho^{(3)}\right) \hat{F}(u)+O\left(g^{4}\right)=\ln F_{q}(u) \\
+ & {\left[\frac{1}{3 !} \sum_{123} u_{1} u_{2} u_{3} \operatorname{Sp}\left(\rho^{(2)}+\rho^{(3)}\right) \hat{\xi}_{1} \hat{\xi}_{2} \hat{\xi}_{3}+\frac{1}{4 !} \sum_{1234} u_{1} u_{2} u_{3} u_{4} \operatorname{Sp}\left(\rho^{(2)}+\rho^{(3)}\right)\right.} \\
& \left.\times \hat{\xi}_{1} \hat{\xi}_{2} \hat{\xi}_{3} \hat{\xi}_{4}+O\left(g^{2} u^{5}, g^{3} u^{5}, g^{4}\right)\right]\left[1+\sum_{5} u_{5} \xi_{5}+O\left(u^{2}\right)\right]^{-1},
\end{aligned}
$$

$\left(\sum_{i} \equiv \sum_{\mu_{i}}\right)$ because $\operatorname{Sp} \rho^{(s)} \hat{\xi}_{1}=0, \operatorname{Sp} \rho^{(s)} \hat{\xi}_{1} \hat{\xi}_{2}=0$ for $s \geqslant 1$ (see (20), (22)). This relation leads to the following expressions for correlations

$$
\begin{aligned}
g_{123}\left(\xi, g_{2}\right) & =\operatorname{Sp}\left(\rho^{(2)}+\rho^{(3)}\right) \hat{\xi}_{1} \hat{\xi}_{2} \hat{\xi}_{3}+O\left(g^{4}\right) \sim g^{2} \\
g_{1234}\left(\xi, g_{2}\right) & =\operatorname{Sp}\left(\rho^{(2)}+\rho^{(3)}\right)\left[\hat{\xi}_{1} \hat{\xi}_{2} \hat{\xi}_{3} \hat{\xi}_{4}-\left(\hat{\xi}_{1} \hat{\xi}_{2} \hat{\xi}_{3} \xi_{4}+c . p .\right)\right]+O\left(g^{4}\right) \sim g^{2} .
\end{aligned}
$$

Taking into account these estimates and formula (69) we obtain an average current for $\mathrm{FED}_{2}$

$$
\begin{aligned}
j_{1 \mu}\left(\xi, g_{2}\right)= & \sum_{\mu_{1}} \sigma_{\mu, \mu_{1}} \xi_{\mu_{1}}+\sum_{\mu_{1}, \mu_{2}} \sigma_{\mu_{, 1} \mu_{2}}\left(\xi_{\mu_{1}} \xi_{\mu_{2}}+\delta g_{\mu_{1} \mu_{2}}\right) \\
& +\sum_{\mu_{1}, \mu_{2}, \mu_{3}} \sigma_{\mu, \mu_{1} \mu_{2} \mu_{3}}\left(\xi_{\mu_{1}} \xi_{\mu_{2}}+3 g_{\mu_{1} \mu_{2}}\right) \xi_{\mu_{3}}+O\left(g^{5}\right)
\end{aligned}
$$

and the averaged electromagnetic field $\xi_{\mu}(t)$ satisfies the Maxwell equation

$$
\partial_{t} \xi_{\mu}=\mathrm{i} \sum_{\mu^{\prime}} c_{\mu \mu^{\prime}} \xi_{\mu^{\prime}}-4 \pi j_{1 \mu}\left(\xi, g_{2}\right)
$$

(see (52)). According to (49), (52), (58) time equation of the FED for the binary correlations has the form

$$
\begin{aligned}
\partial_{t} g_{\mu \mu^{\prime}}= & \mathrm{i} \sum_{\mu^{\prime \prime}} c_{\mu \mu^{\prime \prime}} g_{\mu^{\prime \prime} \mu^{\prime}}-4 \pi e^{G\left(g, \frac{\partial}{\partial \xi}\right)}\left(G_{\mu}\left(g, \frac{\partial}{\partial \xi}\right)+4 \pi T \frac{\partial}{\partial \xi_{\mu}}\right) \\
& \left.\times I_{\mu^{\prime}}(u=0, \xi)+\left(\mu \leftrightarrow \mu^{\prime}\right)\right) .
\end{aligned}
$$

Now using formulae (45), (49), (61) and estimates (73) we obtain the following equation of the $\mathrm{FED}_{2}$ for binary correlations

$$
\begin{aligned}
\partial_{t} g_{\mu \mu^{\prime}}= & i \sum_{\mu^{\prime \prime}} c_{\mu \mu^{\prime \prime}} g_{\mu^{\prime \prime} \mu^{\prime}}-4 \pi \sum_{\mu^{\prime \prime}}\left(g_{\mu^{\prime \prime} \mu^{\prime}}+4 \pi T \delta_{\mu^{\prime \prime} \mu^{\prime}}\right) \\
& \times\left(\sigma_{\mu, \mu^{\prime \prime}}+2 \sum_{\mu_{1}} \sigma_{\mu, \mu^{\prime \prime} \mu_{1}} \xi_{\mu_{1}}+3 \sum_{\mu_{1}, \mu_{2}} \sigma_{\mu, \mu^{\prime \prime} \mu_{1} \mu_{2}}\left(\xi_{\mu_{1}} \xi_{\mu_{2}}+g_{\mu_{1} \mu_{2}}\right)\right) \\
& +\left(\mu \leftrightarrow \mu^{\prime}\right)+O\left(g^{5}\right)
\end{aligned}
$$


Expressions (74), (75), (77) give equations of the $\mathrm{FED}_{2}$, i.e. the equations of the FED taking into account only binary correlations.

Note finally that we cannot build the NED similar to our consideration of $\mathrm{FED}_{2}$ because quasi-equilibrium distribution $\rho_{q}(22)$ does not exist. This probably indicates that a reduced description of electromagnetic field only by average electric and magnetic fields in a general case is impossible (see also [10]).

In the present work the classical FED is considered. This theory is a limit of the corresponding quantum theory. In a quantum case the binary correlation function $g_{\mu_{1} \mu_{2}}$ is closely related to the Wigner photon distribution functions. Therefore, equation (77) is similar to the kinetic equation for photons in which square in $g_{\mu_{1} \mu_{2}}$ terms describe the photon-photon collisions in the medium.

\section{Conclusions}

Thus, there has been built a fluctuation electrodynamics of classical electromagnetic field in equilibrium medium as a theory which describes its nonequilibrium states by average field and all field correlations. Equations of the fluctuation electrodynamics are written in terms of a generating functional for moments of the field and in terms of an average field and a generating functional for correlations (fluctuations, centered moments). It was established that the right hand sides of the corresponding equations are completely defined by an average electric current calculated in the present paper up to the fourth order in electromagnetic interaction. The dependence of the current on electromagnetic field and its correlations (the material equation of the theory) is nonlinear, local in time and nonlocal in space (spatial dispersion). The paper discusses the relation of the developed theory to the description of the electromagnetic field with average field and its binary correlations.

This work was supported by the State Foundation for Fundamental Research of Ukraine (project No. 2.7/418) and INTAS (project No. 00-577).

\section{References}

1. Kilin S.Ya. Quantum Optics. Fields and their Detecting. Navuka i Tekhnika, Minsk, 1990 (in Russian).

2. Klimontovich Yu.L. Kinetic Theory of Nonideal Gases and Nonideal Plasmas. Nauka, Moscow, 1975 (in Russian); Pergamon Press, Oxford, 1982.

3. Balescu R. Statistical Mechanics of Charged Particles. John Wiley and Sons, London, 1963.

4. Bogolyubov N.N. Problems of Dynamical Theory in Statistical Physics. Gostekhizdat, Moscow, 1946 (in Russian).

5. Akhiezer A.I., Peletmisky S.V. Methods of Statistical Physics. Nauka, Moscow, 1977 (in Russian); Pergamon Press, Oxford, 1981.

6. Peletmisky S.V., Sokolovsky A.I., General equations of fluctuation hydrodynamics. Ukrainian Journal of Physics, 1992, 37, 1521-1528 (in Russian).

7. Alexandrov A.F., Bogdankevich L.S., Rukhadze A.A. Foundations of Plasma Electrodynamics. Vysshaya Shkola, Moscow, 1988 (in Russian). 
8. Sokolovsky A.I., Stupka A.A., Kinetic theory of electromagnetic processes in equilibrium medium. Visnyk Dnipropetrovs'kogo Natsional'nogo Universytetu. Physics, Radio Electronics, 2003, 10, 63-70 (in Ukrainian).

9. Sokolovsky A.I., Stupka A.A., Modes of electromagnetic field in equilibrium plasma. The Journal of Kharkiv National University. "Nuclei, Paryicles, Fields", 2004, No. 628, 2(24), 87-92 (in Ukrainian).

10. Peletminskii S.V., Prikhod'ko V.I., Schelokov V.S., Low-frequency asymptotics of elecrodynamic Green functions. Teoreticheskaya i matematicheskaya fizika, 1975, 25, No. 1, 70-79 (in Russian).

\title{
Класична флуктуаційна електродинаміка
}

\author{
О.Й.Соколовський, А.А. Ступка
}

Дніпропетровський національний університет, вул. Наукова 13, Дніпропетровськ, Україна, 49050

Отримано 3 серпня 2005 р., в остаточному вигляді - 8 листопада 2005 р.

Система, яка складається з рівноважного середовища із заряджених частинок і електромагнітного поля, розглянута в класичному випадку та при малій взаємодії між підсистемами. Функцію розподілу системи розраховано з точністю до третього порядку за електромагнітною взаємодією. Поле описується усіма моментами електричного та магнітного поля, які обрані параметрами скороченого опису в рамках метода скороченого опису нерівноважних станів Боголюбова. Як гранична умова до рівняння Ліувілля застосована узагальнена умова Боголюбова повного ослаблення кореляцій між підсистемами. Одержані часові рівняння для параметрів скороченого опису записані в компактній формі за допомогою породжуючого функціоналу для моментів поля та породжуючого функціоналу для кореляцій поля (центрованих моментів, флуктуацій). Отримані рівняння узагальнюють нелінійну електродинаміку в рівноважному середовищі на випадок врахування флуктуацій електроманітного поля.

Ключові слова: метод скороченого опису нерівноважних станів Боголюбова, узагальнена гранична умова повного ослаблення кореляцій, флуктуаційна електродинаміка у рівноважному середовищі, породжуючий функціонал

PACS: 05.20.-y, 05.40.-a, 11., 52.40.Db 